\title{
Iterative Coupling for Fully Dynamic Poroelasticity
}

\author{
M. Bause, J. W. Both ${ }^{\dagger}$, F. A. Radu ${ }^{\ddagger}$ \\ * Helmut Schmidt University, Faculty of Mechanical Engineering, Holstenhofweg 85, \\ 22043 Hamburg, Germany \\ $\dagger, \ddagger$ University of Bergen, Department of Mathematics, Allégaten 41, \\ 50520 Bergen, Norway
}

\begin{abstract}
We present an iterative coupling scheme for the numerical approximation of the mixed hyperbolic-parabolic system of fully dynamic poroelasticity. We prove its convergence in the Banach space setting for an abstract semi-discretization in time that allows the application of the family of diagonally implicit Runge-Kutta methods. Recasting the semi-discrete solution as the minimizer of a properly defined energy functional, the proof of convergence uses its alternating minimization. The scheme is closely related to the undrained split for the quasi-static Biot system.
\end{abstract}

Keywords. Fully dynamic poroelasticity, Biot-Allard equations, iterative coupling, splitting scheme, convergence.

2010 Mathematics Subject Classification. Primary 65M12. Secondary 76S05.

\section{Introduction}

Information on flow in deformable porous media has become of increasing importance in various fields of natural sciences and technology. It offers an abundance of technical, geophysical, environmental and biomedical applications including modern material science polymers and metal foams, gaining significance particularly in lightweight design and aircraft industry, design of batteries or hydrogen fuel cells for green technologies, geothermal energy exploration or reservoir engineering as well as mechanism in the human body and food technology. Consequently, quantitative methods, based on numerical simulations, are desirable in analyzing experimental data and designing theories based on mathematical concepts. Recently, the quasi-static Biot system (cf., e.g., [12, 14]) has attracted researcher's interest and has been studied as a proper model for the numerical simulation of flow in deformable porous media. The design, analysis and optimization of approximation techniques that are based on an iterative coupling of the subproblems of fluid flow and mechanical deformation were focused strongly.

\footnotetext{
*bause@hsu-hh.de (corresponding author), ${ }^{\dagger}$ Jakub.Both@uib.no, ${ }^{\ddagger}$ Florin.Radu@uib.no
} 
Iterative coupling offers the appreciable advantage over the fully coupled method that existing and highly developed discretizations and algebraic solver technologies can be reused. For the quasi-static Biot system, pioneering work is done in [10, 12. Further research is presented in, e.g., [2, 4, 7, 8, 9, 13].

In the case of larger contrast coefficients that stand for the ratio between the intrinsical characteristic time and the characteristic domain time scale the fully dynamic hyperbolic-parabolic system of poroelasticity has to be considered. In [11, this system (referred to as the BiotAllard equations) is derived by asymptotic homogenization in the space and time variables. Here, to fix our ideas and carve out the key technique of proof, a simplified form of the system proposed in 11 is studied. However, its mixed hyperbolic-parabolic structure is preserved. Our modification of the fully dynamic poroelasticity model in [1] comes through a simplication of the solution's convolution with the dynamic permeability that is defined as the spatial average of pore system Stokes solutions on the unit cell. The fully dynamic system of poroelasticity to be analyzed here is given by (cf. [14, p. 313])

$$
\begin{aligned}
\rho \partial_{t}^{2} \boldsymbol{u}-\nabla \cdot(\mathbb{C} \varepsilon(\boldsymbol{u})-\boldsymbol{\alpha} p) & =\boldsymbol{f}, \\
\partial_{t}\left(c_{0} p+\boldsymbol{\alpha}: \varepsilon(\boldsymbol{u})\right)+\nabla \cdot \boldsymbol{q} & =h, \\
\boldsymbol{\kappa}^{-1} \boldsymbol{q}+\nabla p & =\boldsymbol{g} .
\end{aligned}
$$

System (1.1) is equiped with appropriate initial and boundary conditions. In (1.1), the variable $\boldsymbol{u}$ is the unkown effective solid phase displacement and $p$ is the unkown effective pressure. The quantity $\boldsymbol{\varepsilon}(\boldsymbol{u})=\left(\nabla \boldsymbol{u}+(\nabla \boldsymbol{u})^{\top}\right) / 2$ denotes the symmetrized gradient or strain tensor. Further, $\rho$ is the effective mass density, $\mathbb{C}$ is Gassmann's fourth order effective elasticity tensor, $\boldsymbol{\alpha}$ is Biot's pressure-storage coupling tensor and $c_{0}$ is the specific storage coefficient. In the three field formulation (1.1), the vector field $\boldsymbol{q}$ is Darcy's velocity and $\boldsymbol{\kappa}$ is the permeability tensor. All tensors are assumed to be symmetric, bounded and uniformly positive definite, the constants $\rho$ and $c_{0}$ are positive. By $\boldsymbol{A}: \boldsymbol{B}$ we denote the Frobenius inner product of $\boldsymbol{A}$ and $\boldsymbol{B}$. The functions on the right-hand side of (1.1) are supposed to be elements in dual spaces and, therefore, can include body forces and surface data (boundary conditions).

So far, the numerical simulation of the system (1.1) has been studied rarely in the literature despite its numerous applications in practice. This might be due to the mixed hyberbolicparabolic character of the system and severe complexities involved in the construction of monolithic solver or iterative coupling schemes with guaranteed stability properties. Spacetime finite element approximations of hyperbolic and parabolic problems and the quasi-static Biot system were recently proposed, analyzed and investigated numerically by the authors in [1, 2, 3. Here, we propose an iterative coupling scheme for the system (1.1) and prove its convergence. This is done in Banach spaces for the semi-discretization in time of (1.1). An abstract setting is used for the time discretization such that the family of diagonally implicit Runge-Kutta methods becomes applicable. The key ingredient of our proof of convergence is the observation that we can recast the semi-discrete approximation of (1.1) as the minimizer of an energy functional in the displacement and Darcy velocity fields. To solve the minimization problem, the general and abstract framework of alternating minimization (cf. [5, 6]) is applied. The resulting subproblems of this minimization are then reformulated as our final iterative coupling scheme. Thereby, the proof of convergence of the iterative scheme is traced back to the convergence of the alternating minimization approach. This shows that the latter provides an abstract and powerful tool of optimization for the design of iterative coupling schemes. 
We use standard notation. In particular, we denote by $\langle\cdot, \cdot\rangle$ the standard inner product of $L^{2}(\Omega)$ and by $\|\cdot\|$ the norm of $L^{2}(\Omega)$.

\section{Variational formulation of a semi-discrete approximation of the system of dynamic poroelasticity}

Firstly, we discretize the continuous system of dynamic poroelasticity (1.1) in time by using arbitrary (diagonally implicit) Runge-Kutta methods and formulate the semi-discrete approximation as solution to a minimization problem, following the approach in [5]. For this, we consider an equidistant partition $0=t_{0}<t_{1}<\ldots<t_{N}=T$ of the time interval of interest $[0, T]$ with time step size $\Delta t$. In the sequel, we use the following function spaces for displacement, pressure, and flux, respectively,

$$
\begin{aligned}
\boldsymbol{V}^{n} & :=\left\{\boldsymbol{v} \in H^{1}(\Omega)^{d} \mid \boldsymbol{v}^{n} \text { satisfies prescribed BC at time } t_{n}\right\}, \\
Q^{n} & :=L^{2}(\Omega), \\
\boldsymbol{W}^{n} & :=\left\{\boldsymbol{w} \in H(\operatorname{div} ; \Omega) \mid \boldsymbol{w} \text { satisfies prescribed BC at time } t_{n}\right\} .
\end{aligned}
$$

Further, let $\boldsymbol{V}_{0}, Q_{0}$, and $\boldsymbol{W}_{0}$ denote the corresponding natural test spaces, and $\boldsymbol{V}_{0}^{\star}, Q_{0}^{\star}$, and $\boldsymbol{W}_{0}^{\star}$ their dual spaces.

Applying any diagonally implicit Runge-Kutta method for the temporal discretization of (1.1), eventually involves solving systems of the following structure.

Problem 2.1 In the $n$-th time step, find the displacement $\boldsymbol{u}^{n} \in \boldsymbol{V}^{n}$, pressure $p^{n} \in Q^{n}$, and flux $\boldsymbol{q}^{n} \in \boldsymbol{W}^{n}$, satisfying for all $(\boldsymbol{v}, q, \boldsymbol{w}) \in \boldsymbol{V}_{0} \times Q_{0} \times \boldsymbol{W}_{0}$ the equations

$$
\begin{aligned}
\frac{\rho}{\Delta t^{2}}\left\langle\boldsymbol{u}^{n}, \boldsymbol{v}\right\rangle+\theta_{1}\left\langle\mathbb{C} \varepsilon\left(\boldsymbol{u}^{n}\right), \boldsymbol{\varepsilon}(\boldsymbol{v})\right\rangle-\left\langle\boldsymbol{\alpha} p^{n}, \boldsymbol{\varepsilon}(\boldsymbol{v})\right\rangle & =\left\langle\boldsymbol{f}_{\boldsymbol{\theta}, \Delta t}^{n}, \boldsymbol{v}\right\rangle \\
c_{0}\left\langle p^{n}, q\right\rangle+\left\langle\boldsymbol{\alpha}: \varepsilon\left(\boldsymbol{u}^{n}\right), q\right\rangle+\theta_{2} \Delta t\left\langle\nabla \cdot \boldsymbol{q}^{n}, q\right\rangle & =\left\langle h_{\boldsymbol{\theta}, \Delta t}^{n}, q\right\rangle \\
\left\langle\boldsymbol{\kappa}^{-1} \boldsymbol{q}^{n}, \boldsymbol{w}\right\rangle-\left\langle p^{n}, \nabla \cdot \boldsymbol{w}\right\rangle & =\left\langle\boldsymbol{g}_{\boldsymbol{\theta}, \Delta t}^{n}, \boldsymbol{w}\right\rangle .
\end{aligned}
$$

In (2.1), the quantities $\theta_{1}, \theta_{2} \in(0,1]$ are discretization parameters, and the right-hand side functions $\boldsymbol{f}_{\boldsymbol{\theta}, \Delta t}^{n} \in \boldsymbol{V}_{0}^{\star}, h_{\boldsymbol{\theta}, \Delta t}^{n} \in Q_{0}^{\star}, \boldsymbol{g}_{\boldsymbol{\theta}, \Delta t}^{n} \in \boldsymbol{W}_{0}^{\star}$ include information on external volume and surface terms, as well as previous time steps depending on the choice of the implicit RungeKutta discretization.

Assuming positive compressibility, i.e., $c_{0}>0$ for the specific storage coefficient, the semidiscrete approximation satisfies equivalently the following variational problem; cf. [5] for the derivation of a similar equivalence in the framework of the quasi-static Biot system.

Problem 2.2 Find $\left(\boldsymbol{u}^{n}, \boldsymbol{q}^{n}\right) \in \boldsymbol{V}^{n} \times \boldsymbol{W}^{n}$, satisfying

$$
\left(\boldsymbol{u}^{n}, \boldsymbol{q}^{n}\right)=\underset{(\boldsymbol{u}, \boldsymbol{q}) \in \boldsymbol{V}^{n} \times \boldsymbol{W}^{n}}{\arg \min } \mathcal{E}(\boldsymbol{u}, \boldsymbol{q})
$$


where the energy $\mathcal{E}: \boldsymbol{V}^{n} \times \boldsymbol{W}^{n} \rightarrow \mathbb{R}$ at time $t_{n}$ is defined by $\left((\boldsymbol{u}, \boldsymbol{q}) \in \boldsymbol{V}^{n} \times \boldsymbol{W}^{n}\right)$

$$
\begin{aligned}
\mathcal{E}(\boldsymbol{u}, \boldsymbol{q}):= & \frac{\rho}{2 \Delta t^{2}}\|\boldsymbol{u}\|^{2}+\frac{\theta}{2}\langle\mathbb{C} \boldsymbol{\varepsilon}(\boldsymbol{u}), \boldsymbol{\varepsilon}(\boldsymbol{u})\rangle+\frac{\theta_{1} \theta_{2} \Delta t}{2}\left\langle\boldsymbol{\kappa}^{-1} \boldsymbol{q}, \boldsymbol{q}\right\rangle \\
& \quad+\frac{\theta_{1}}{2 c_{0}}\left\|h_{\boldsymbol{\theta}, \Delta t}^{n}-\boldsymbol{\alpha}: \boldsymbol{\varepsilon}(\boldsymbol{u})-\theta_{2} \Delta t \nabla \cdot \boldsymbol{q}\right\|^{2}-\left\langle\boldsymbol{f}_{\boldsymbol{\theta}, \Delta t}^{n}, \boldsymbol{u}\right\rangle-\left\langle\boldsymbol{g}_{\boldsymbol{\theta}, \Delta t}^{n}, \boldsymbol{q}\right\rangle .
\end{aligned}
$$

The semi-discrete pressure $p^{n}$ may then be recovered by the post-processing step

$$
p^{n}=c_{0}^{-1}\left(h_{\boldsymbol{\theta}, \Delta t}^{n}-\boldsymbol{\alpha}: \varepsilon\left(\boldsymbol{u}^{n}\right)-\theta_{2} \Delta t \nabla \cdot \boldsymbol{q}^{n}\right) .
$$

\section{Iterative coupling for the system of dynamic poroelasticity}

Following the philosophy of [5], we propose an iterative coupling of the semi-discrete equations (2.1) of dynamic poroelasticity by firstly applying the fundamental alternating minimization to the variational formulation (2.2); cf. Alg. 3.1.

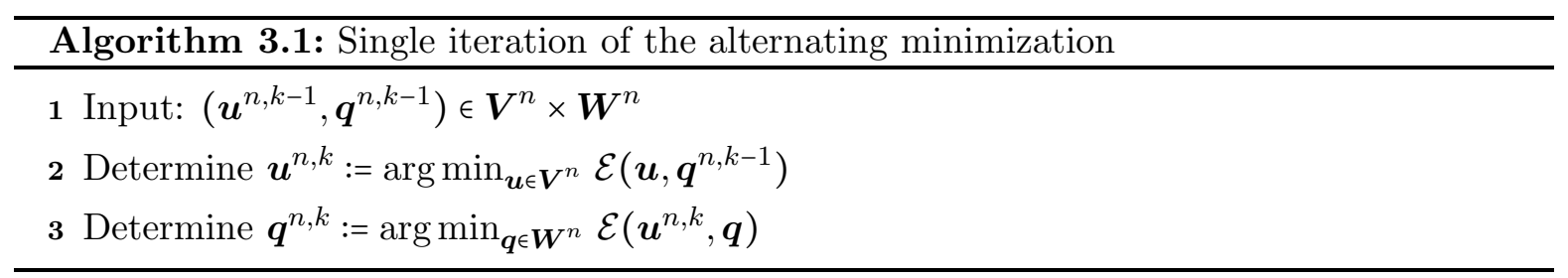

Secondly, the resulting scheme is equivalently reformated in terms of a stabilized splitting scheme applied to the three-field formulation (2.1). For this, a pressure iterate $p^{n, k}=c_{0}^{-1}\left(h_{\boldsymbol{\theta}, \Delta t}^{n}\right.$ $\left.-\boldsymbol{\alpha}: \boldsymbol{\varepsilon}\left(\boldsymbol{u}^{n, k}\right)-\theta_{2} \Delta t \nabla \cdot \boldsymbol{q}^{n, k}\right) \in Q^{n}, k \geq 0$, is introduced, consistent with (2.4), and the optimality conditions corresponding to the two steps of Alg. 3.1 are reformulated. The calculations are skipped here. We immediately present the resulting scheme, which in the end is closely related to the undrained split for the quasi-static Biot system [10].

Problem 3.1 Let $\left(\boldsymbol{u}^{n, 0}, p^{n, 0}\right) \in \boldsymbol{V}^{n} \times Q^{n}$ be given and $k \geq 1$.

1. Step (Update of mechanical deformation): For given $\left(\boldsymbol{u}^{n, k-1}, p^{n, k-1}\right) \in \boldsymbol{V}^{n} \times Q^{n}$, find $\boldsymbol{u}^{n, k} \in$ $\boldsymbol{V}^{n}$ satisfying for all $\boldsymbol{v} \in \boldsymbol{V}_{0}$,

$$
\begin{gathered}
\frac{\rho}{\Delta t^{2}}\left\langle\boldsymbol{u}^{n, k}, \boldsymbol{v}\right\rangle+\theta_{1}\left\langle\mathbb{C} \boldsymbol{\varepsilon}\left(\boldsymbol{u}^{n, k}\right)+\frac{\boldsymbol{\alpha} \otimes \boldsymbol{\alpha}}{c_{0}} \boldsymbol{\varepsilon}\left(\boldsymbol{u}^{n, k}-\boldsymbol{u}^{n, k-1}\right), \boldsymbol{\varepsilon}(\boldsymbol{v})\right\rangle \\
-\theta_{1}\left\langle\boldsymbol{\alpha} p^{n, k-1}, \boldsymbol{\varepsilon}(\boldsymbol{v})\right\rangle=\left\langle\boldsymbol{f}_{\boldsymbol{\theta}, \Delta t}^{n}, \boldsymbol{v}\right\rangle,
\end{gathered}
$$

where $\otimes: \mathbb{R}^{d \times d} \times \mathbb{R}^{d \times d} \rightarrow \mathbb{R}^{d \times d \times d \times d}$ denotes the standard tensor product.

2. Step (Update of Darcy velocity and pressure): For given $\left(\boldsymbol{u}^{n, k}, p^{n, k-1}\right) \in \boldsymbol{V}^{n} \times Q^{n}$ find $\left(p^{n, k}, \boldsymbol{q}^{n, k}\right) \in Q^{n} \times \boldsymbol{W}^{n}$ satisfying for all $(q, \boldsymbol{w}) \in Q_{0} \times \boldsymbol{W}_{0}$,

$$
\begin{aligned}
c_{0}\left\langle p^{n, k}, q\right\rangle+\left\langle\boldsymbol{\alpha}: \boldsymbol{\varepsilon}\left(\boldsymbol{u}^{n, k}\right), q\right\rangle+\theta_{2} \Delta t\left\langle\nabla \cdot \boldsymbol{q}^{n, k}, q\right\rangle & =\left\langle h_{\boldsymbol{\theta}, \Delta t}^{n}, q\right\rangle, \\
\left\langle\boldsymbol{\kappa}^{-1} \boldsymbol{q}^{n, k}, \boldsymbol{w}\right\rangle-\left\langle p^{n, k}, \nabla \cdot \boldsymbol{w}\right\rangle & =\left\langle\boldsymbol{g}_{\boldsymbol{\theta}, \Delta t}^{n}, \boldsymbol{w}\right\rangle .
\end{aligned}
$$

We note that the splitting scheme defined by (3.1), (3.2) utilizes the identical stabilization as the undrained split for the quasi-static Biot equations [10]. 


\section{Convergence of the iterative coupling scheme}

The identification of the undrained split approach (3.1), (3.2) as the application of the alternating minimization, cf. Alg. 3.1, to the variational problem (2.2) yields the basis for a simple convergence analysis. For this, we utilize the following abstract convergence result, that is rewritten here in terms of the specific formulation of Alg. 3.1.

Theorem 4.1 (Convergence of the alternating minimization [6]) Let $|\cdot|,|\cdot|_{\mathrm{m}}$, and $\mid \cdot$ If denote semi-norms on $\boldsymbol{V}_{0} \times \boldsymbol{W}_{0}, \boldsymbol{V}_{0}$, and $\boldsymbol{W}_{0}$, respectively. Let $\beta_{\mathrm{m}}, \beta_{\mathrm{f}} \geq 0$ satisfy the inequalities

$$
|(\boldsymbol{v}, \boldsymbol{w})|^{2} \geq \beta_{\mathrm{m}}|\boldsymbol{v}|_{\mathrm{m}}^{2} \quad \text { and } \quad|(\boldsymbol{v}, \boldsymbol{w})|^{2} \geq \beta_{\mathrm{f}}|\boldsymbol{w}|_{\mathrm{f}}^{2}
$$

for all $(\boldsymbol{v}, \boldsymbol{w}) \in \boldsymbol{V}_{0} \times \boldsymbol{W}_{0}$. Furthermore, assume that the energy functional $\mathcal{E}$ of (2.3) satisfies the following conditions:

- The energy $\mathcal{E}$ is Frechét differentiable with DE denoting its derivative.

- The energy $\mathcal{E}$ is strongly convex wrt. $|\cdot|$ with modulus $\sigma>0$, i.e., for all $\boldsymbol{u}, \overline{\boldsymbol{u}} \in \boldsymbol{V}^{n}$ and $\boldsymbol{q}, \overline{\boldsymbol{q}} \in \boldsymbol{W}^{n}$ it holds that

$$
\mathcal{E}(\overline{\boldsymbol{u}}, \overline{\boldsymbol{q}}) \geq \mathcal{E}(\boldsymbol{u}, \boldsymbol{q})+\langle D \mathcal{E}(\boldsymbol{u}, \boldsymbol{q}),(\overline{\boldsymbol{u}}-\boldsymbol{u}, \overline{\boldsymbol{q}}-\boldsymbol{q})\rangle+\frac{\sigma}{2}|(\overline{\boldsymbol{u}}-\boldsymbol{u}, \overline{\boldsymbol{q}}-\boldsymbol{q})|^{2} .
$$

- The partial functional derivatives $D_{\boldsymbol{u}} \mathcal{E}$ and $D_{q} \mathcal{E}$ are uniformly Lipschitz continuous wrt. $|\cdot|_{\mathrm{m}}$ and $|\cdot|_{\mathrm{f}}$ with Lipschitz constants $L_{\mathrm{m}}$ and $L_{\mathrm{f}}$, respectively, i.e., for all $(\boldsymbol{u}, \boldsymbol{q}) \in \boldsymbol{V}^{n} \times \boldsymbol{W}^{n}$ and $(\boldsymbol{v}, \boldsymbol{w}) \in \boldsymbol{V}_{0} \times \boldsymbol{W}_{0}$ it holds that

$$
\begin{gathered}
\mathcal{E}(\boldsymbol{u}+\boldsymbol{v}, \boldsymbol{q}) \leq \mathcal{E}(\boldsymbol{u}, \boldsymbol{q})+\left\langle D_{\boldsymbol{u}} \mathcal{E}(\boldsymbol{u}, \boldsymbol{q}), \boldsymbol{v}\right\rangle+\frac{L_{\mathrm{m}}}{2}\|\boldsymbol{v}\|_{\mathrm{m}}^{2}, \\
\mathcal{E}(\boldsymbol{u}, \boldsymbol{q}+\boldsymbol{w}) \leq \mathcal{E}(\boldsymbol{u}, \boldsymbol{q})+\left\langle D_{\boldsymbol{q}} \mathcal{E}(\boldsymbol{u}, \boldsymbol{q}), \boldsymbol{w}\right\rangle+\frac{L_{\mathrm{f}}}{2}\|\boldsymbol{w}\|_{\mathrm{f}}^{2} .
\end{gathered}
$$

Let $\left(\boldsymbol{u}^{n}, \boldsymbol{q}^{n}\right) \in \boldsymbol{V}^{n} \times \boldsymbol{W}^{n}$ denote the solution to (2.2), and let $\left(\boldsymbol{u}^{n, k}, \boldsymbol{q}^{n, k}\right)$ denote the corresponding approximation defined by Alg. 3.1. Then, for all $k \geq 1$ it follows that

$$
\begin{aligned}
& \mathcal{E}\left(\boldsymbol{u}^{n, k}, \boldsymbol{q}^{n, k}\right)-\mathcal{E}\left(\boldsymbol{u}^{n}, \boldsymbol{q}^{n}\right) \\
& \quad \leq\left(1-\frac{\beta_{\mathrm{m}} \sigma}{L_{\mathrm{m}}}\right)\left(1-\frac{\beta_{\mathrm{f}} \sigma}{L_{\mathrm{f}}}\right)\left(\mathcal{E}\left(\boldsymbol{u}^{n, k-1}, \boldsymbol{q}^{n, k-1}\right)-\mathcal{E}\left(\boldsymbol{u}^{n}, \boldsymbol{q}^{n}\right)\right) .
\end{aligned}
$$

A simple application of Theorem 4.1 now yields the main result of the work, namely the global linear convergence of the undrained split (3.1), (3.2).

Corollary 4.2 (Linear convergence of the undrained split) Let $|\cdot|$ be defined by

$$
\begin{gathered}
|(\boldsymbol{v}, \boldsymbol{w})|^{2}:=\frac{\rho}{\Delta t^{2}}\|\boldsymbol{v}\|^{2}+\theta_{1}\langle\mathbb{C} \varepsilon(\boldsymbol{v}), \varepsilon(\boldsymbol{v})\rangle+\theta_{1} \theta_{2} \Delta t\left\langle\boldsymbol{\kappa}^{-1} \boldsymbol{w}, \boldsymbol{w}\right\rangle \\
+\frac{\theta_{1}}{c_{0}}\left\|\boldsymbol{\alpha}: \boldsymbol{\varepsilon}(\boldsymbol{v})+\theta_{2} \Delta t \nabla \cdot \boldsymbol{w}\right\|^{2}
\end{gathered}
$$


for all $(\boldsymbol{v}, \boldsymbol{w}) \in \boldsymbol{V}_{0} \times \boldsymbol{W}_{0}$. Furthermore, let $\left(\boldsymbol{u}^{n}, \boldsymbol{q}^{n}\right) \in \boldsymbol{V}^{n} \times \boldsymbol{W}^{n}$ denote the solution to (2.2), and let $\left(\boldsymbol{u}^{n, k}, \boldsymbol{q}^{n, k}\right) \in \boldsymbol{V}^{n} \times \boldsymbol{W}^{n}$ denote the corresponding approximation defined by Alg. 3.1. Then, for all $k \geq 1$ it holds that

$$
\begin{aligned}
\left|\left(\boldsymbol{u}^{n, k}-\boldsymbol{u}^{n}, \boldsymbol{q}^{n, k}-\boldsymbol{q}^{n}\right)\right|^{2} \leq & \left.\frac{\left\|\boldsymbol{\alpha}: \mathbb{C}^{-1}: \boldsymbol{\alpha}\right\|_{\infty}}{c_{0}+\left\|\boldsymbol{\alpha}: \mathbb{C}^{-1}: \boldsymbol{\alpha}\right\|_{\infty}}\right)^{2} \\
& \cdot\left|\left(\boldsymbol{u}^{n, k-1}-\boldsymbol{u}^{n}, \boldsymbol{q}^{n, k-1}-\boldsymbol{q}^{n}\right)\right|^{2} .
\end{aligned}
$$

Proof. We first examine convexity and smoothness properties of $\mathcal{E}$ defined in (2.3) by analyzing the second functional derivative of $\mathcal{E}$. For this, let $(\boldsymbol{u}, \boldsymbol{q}) \in \boldsymbol{V}^{n} \times \boldsymbol{W}^{n}$ and $(\boldsymbol{v}, \boldsymbol{w}) \in \boldsymbol{V}_{0} \times \boldsymbol{W}_{0}$ be arbitrary. Then, for the second functional derivative $D^{2} \mathcal{E}(\boldsymbol{u}, \boldsymbol{q}):\left(\boldsymbol{V}_{0}^{\star} \times \boldsymbol{W}_{0}^{\star}\right)^{2} \rightarrow \mathbb{R}$ of $\mathcal{E}$ it holds that

$$
\left\langle D^{2} \mathcal{E}(\boldsymbol{u}, \boldsymbol{q})(\boldsymbol{v}, \boldsymbol{w}),(\boldsymbol{v}, \boldsymbol{w})\right\rangle=|(\boldsymbol{v}, \boldsymbol{w})|^{2} .
$$

Next, we define a norm $|\cdot|_{\mathrm{m}}$ on $\boldsymbol{V}_{0}$ by considering the partial second functional derivative of $\mathcal{E}$ with respect to the displacement field,

$$
\left\langle D_{\boldsymbol{u}}^{2} \mathcal{E}(\boldsymbol{u}, \boldsymbol{q}) \boldsymbol{v}, \boldsymbol{v}\right\rangle=\frac{\rho}{\Delta t^{2}}\|\boldsymbol{v}\|^{2}+\theta_{1}\langle\mathbb{C} \varepsilon(\boldsymbol{v}), \boldsymbol{\varepsilon}(\boldsymbol{v})\rangle+\frac{\theta_{1}}{c_{0}}\|\boldsymbol{\alpha}: \varepsilon(\boldsymbol{v})\|^{2}=:|\boldsymbol{v}|_{\mathrm{m}}^{2} .
$$

Similarly, we define a norm $|\cdot|_{\mathrm{f}}$ on $\boldsymbol{W}_{0}$ by means of

$$
\left\langle D_{\boldsymbol{q}}^{2} \mathcal{E}(\boldsymbol{u}, \boldsymbol{q}) \boldsymbol{w}, \boldsymbol{w}\right\rangle=\theta_{1} \theta_{2} \Delta t\left\langle\boldsymbol{\kappa}^{-1} \boldsymbol{w}, \boldsymbol{w}\right\rangle+\frac{\theta_{1}}{c_{0}}\left\|\theta_{2} \Delta t \nabla \cdot \boldsymbol{w}\right\|^{2}=:|\boldsymbol{w}|_{\mathrm{f}}^{2} .
$$

It directly follows that $\mathcal{E}$ is strongly convex wrt. $|\cdot|$ with modulus $\sigma=1$, and the partial functional derivatives $D_{\boldsymbol{u}} \mathcal{E}$ and $D_{\boldsymbol{q}} \mathcal{E}$ are uniformly Lipschitz continuous wrt. $|\cdot|_{\mathrm{m}}$ and $|\cdot|_{\mathrm{f}}$ with Lipschitz constants $L_{\mathrm{m}}=1$ and $L_{\mathrm{f}}=1$, respectively.

By the Hölder inequality we deduce that

$$
\begin{aligned}
\|\boldsymbol{\alpha}: \boldsymbol{\varepsilon}(\boldsymbol{v})\|^{2} & =\int_{\Omega}|\boldsymbol{\alpha}: \varepsilon(\boldsymbol{v})|^{2} \mathrm{~d} \boldsymbol{x} \leq \int_{\Omega}\left|\boldsymbol{\alpha}: \mathbb{C}^{-1}: \boldsymbol{\alpha}\right||\varepsilon(\boldsymbol{v}): \mathbb{C}: \varepsilon(\boldsymbol{v})| \mathrm{d} \boldsymbol{x} \\
& \leq\left\|\boldsymbol{\alpha}: \mathbb{C}^{-1}: \boldsymbol{\alpha}\right\|_{\infty}\langle\mathbb{C} \varepsilon(\boldsymbol{v}), \varepsilon(\boldsymbol{v})\rangle .
\end{aligned}
$$

Hence, it follows that

$$
|\boldsymbol{v}|_{\mathrm{m}}^{2} \leq\left(1+\frac{\left\|\boldsymbol{\alpha}: \mathbb{C}^{-1}: \boldsymbol{\alpha}\right\|_{\infty}}{c_{0}}\right)|(\boldsymbol{v}, \boldsymbol{w})|^{2}
$$

On the other hand, applying the triangle inequality and Young's inequality, and balancing the arising constants properly yields that

$$
\begin{aligned}
\frac{\theta_{1}}{c_{0}}\left\|\theta_{2} \Delta t \nabla \cdot \boldsymbol{w}\right\|^{2} \leq \frac{\theta_{1}}{c_{0}}\left(1+\frac{\left\|\boldsymbol{\alpha}: \mathbb{C}^{-1}: \boldsymbol{\alpha}\right\|_{\infty}}{c_{0}}\right)\left\|\theta_{2} \Delta t \nabla \cdot \boldsymbol{w}+\boldsymbol{\alpha}: \varepsilon(\boldsymbol{v})\right\|^{2} \\
+\frac{\theta_{1}}{c_{0}}\left(1+\frac{c_{0}}{\left\|\boldsymbol{\alpha}: \mathbb{C}^{-1}: \boldsymbol{\alpha}\right\|_{\infty}}\right)\|\boldsymbol{\alpha}: \boldsymbol{\varepsilon}(\boldsymbol{v})\|^{2} .
\end{aligned}
$$


Together with (4.3), we also conclude that

$$
|\boldsymbol{w}|_{\mathrm{f}}^{2} \leq\left(1+\frac{\left\|\boldsymbol{\alpha}: \mathbb{C}^{-1}: \boldsymbol{\alpha}\right\|_{\infty}}{c_{0}}\right)|(\boldsymbol{v}, \boldsymbol{w})|^{2} .
$$

Thereby, the assumptions of Theorem 4.1 are fulfilled and (4.1) is ensured with constants $\sigma=$ $L_{\mathrm{m}}=L_{\mathrm{f}}=1$ and $\beta_{\mathrm{m}}=\beta_{\mathrm{f}}=\left(1+\frac{\left\|\boldsymbol{\alpha}: \mathbb{C}^{-1}: \boldsymbol{\alpha}\right\|_{\infty}}{c_{0}}\right)^{-1}$. Finally, the assertion follows directly, since $\mathcal{E}$ is quadratic and $\left(\boldsymbol{u}^{n}, \boldsymbol{q}^{n}\right)$ is a local minimum of $\mathcal{E}$ and $|\cdot|$ relates to the second functional derivative of $\mathcal{E}$ via (4.2). Therefore, we have that $\mathcal{E}\left(\boldsymbol{u}^{n, k}, \boldsymbol{q}^{n, k}\right)-\mathcal{E}\left(\boldsymbol{u}^{n}, \boldsymbol{q}^{n}\right)=2\left|\left(\boldsymbol{u}^{n, k}-\boldsymbol{u}^{n}, \boldsymbol{q}^{n, k}-\boldsymbol{q}^{n}\right)\right|^{2}$ for all $k \geq 0$.

Remark 4.3 (Convergence of $p^{n, k}$ ) The convergence of the sequence of pressures $\left\{p^{n, k}\right\}_{k}$ follows now immediately by a standard inf-sup argument.

Remark 4.4 (Comparison with quasi-static case) The final convergence rate in Corollary 4.2 coincides with the one for the undrained split applied to the quasi-static Biot equations for an homogeneous and isotropic bulk; cf. [12]. In that case, the Biot tensor $\boldsymbol{\alpha}$ reduces to $\alpha \mathbf{I}$ for some constant $\alpha \in(0,1]$, and $\mathbb{C}$ is defined by the Lamé parameters, such that $\boldsymbol{\alpha}: \mathbb{C}^{-1}: \boldsymbol{\alpha}=\frac{\alpha^{2}}{K_{\mathrm{dr}}}$, where $K_{\mathrm{dr}}$ is the drained bulk modulus.

\section{Acknowledgement}

This work was supported by the German Academic Exchange Service (DAAD) under the grant ID 57458510 and by the Research Council of Norway (RCN) under the grant ID 294716.

\section{References}

[1] M. Bause, U. Köcher, F. A. Radu, F. Schieweck, Post-processed Galerkin approximation of improved order for wave equations, Math. Comp., 2019, DOI: 10.1090/mcom/3464, pp. 1-33; arXiv:1803.03005.

[2] M. Bause, F. A. Radu, U. Köcher, Space-time finite element approximation of the Biot poroelasticity system with iterative coupling, Comput. Methods Appl. Mech. Engrg., 320 (2017), pp. 745-768.

[3] M. Bause, F. A. Radu, U. Köcher, Error analysis for discretizations of parabolic problems using continuous finite elements in time and mixed finite elements in space, Numer. Math., 137 (2017), pp. 773-818.

[4] J. W. Both, M. Borregales, J. M. Nordbotten, K. Kundan, F. A. Radu, Robust fixed stress splitting for Biot's equations in heterogeneous media, Appl. Math. Lett., 68, pp. 101-108.

[5] J. W. Both, K. Kundan, J. M. Nordbotten, F. A. Radu, The gradient flow structures of thermo-poro-visco-elasticity, arXiv:1907.03134.

[6] J. W. Both, On the rate of convergence of alternating minimization for non-smooth nonstrongly convex optimization in Banach spaces, arXiv:1911.00404. 
[7] N. Castelletto, J. A. White, H. A. Tchelepi, Accuracy and convergence properties of the fixed-stress iterative solution of two-way coupled poromechanics, Int. J. Num. Anal. Meth. Geomechanics, 39 (2015), pp. 1593-1618.

[8] N. Castelletto, J. A. White, M. Ferronato, Scalable algorithms for three-field mixed finite element coupled poromechanics, J. Comp. Phys., 327 (2016), pp. 894-918.

[9] Q. Hong, J. Kraus, M. Lymbery, F. Philo, Conservative discretizations and parameterrobust preconditioners for Biot and multiple-network flux-based poroelasticity models, Numer-. Linear Algebra Appl., 26 (2019), e2242, pp. 1-25.

[10] J. Kim, H. A. Tchelepi, R. Juanes, Stability and convergence of sequential methods for coupled flow and geomechanics: Drained and undrained splits, Comput. Methods Appl. Mech. Engrg., 200 (2011), pp. 2094-2116.

[11] A. Mikelić, M. F. Wheeler, Theory of the dynamic Biot-Allard equations and their link to the quasi-static Biot system, J. Math. Phys., 53 (2012), pp. 123702:1-15.

[12] A. Mikelić, M. F. Wheeler, Convergence of iterative coupling for coupled flow and geomechanics, Comput. Geosci., 17 (2013), pp. 479-496.

[13] A. Mikelić, B. Wang, M. F. Wheeler, Numerical convergence study of iterative coupling for coupled flow and geomechanics, Comput. Geosci., 18 (2014), pp. 325-341.

[14] R. Showalter, Diffusion in poro-elastic media, J. Math. Anal. Appl., 251 (2000), pp. $310-340$. 